\title{
Periodic Variations in the Wavelength Distributions following Photon Interferences: Analogy with Electron Interferences
}

\author{
Maxime Vabre, ${ }^{1}$ Sylvain Girard, ${ }^{1}$ Hervé Gilles, ${ }^{1}$ Burcu S. Frankland, ${ }^{1}$ Florent Porée, ${ }^{1}$ \\ Philippe Leprince, ${ }^{1}$ Jean-Yves Chesnel, ${ }^{1}$ Raul O. Barrachina, ${ }^{2}$ and François Frémont ${ }^{1}$ \\ ${ }^{1}$ Centre de Recherche sur les Ions, les Matériaux et la Photonique, Unité Mixte Université de Caen-CEA-CNRS-EnsiCaen, \\ 6 Boulevard Maréchal Juin, 14050 Caen Cedex 4, France \\ ${ }^{2}$ Centro Atómico Bariloche and Instituto Balseiro, Comisión Nacional de Energía Atómica and Universidad Nacional de Cuyo, \\ 8400 San Carlos de Bariloche, Río Negro, Argentina
}

Correspondence should be addressed to François Frémont, francois.fremont@ensicaen.fr

Received 5 January 2012; Accepted 30 January 2012

Academic Editors: C. Kosmidis and E. Weigold

Copyright (๑) 2012 Maxime Vabre et al. This is an open access article distributed under the Creative Commons Attribution License, which permits unrestricted use, distribution, and reproduction in any medium, provided the original work is properly cited.

\begin{abstract}
A new interference phenomenon is reported, which has so far not been observed with either matter or light. In a nanometer-sized version of Feynman's famous two-slit "thought" experiment with single electrons, the width of a quasi-monochromatic line has been found to oscillate with the detection angle. Since this experiment resembles the original double-slit experiment by Young with light (1807), photon interferences were investigated in order to determine the wavelength distribution as a function of the position in the interference field. In addition to the well-known oscillating dependence of the intensity with a succession of dark and bright fringes, a periodic dependence with respect to the detection position has also been observed for the width of the wavelength distribution, revealing a larger analogy between electron and photon interferences.
\end{abstract}

\section{Introduction}

In 1923, de Broglie advanced the idea of the wave-like nature of a moving matter particle [1]. Since this hypothesis, the wave nature of the electron has been demonstrated in numerous experiments, by observing diffraction or interference patterns resulting in electron scattering on matter, for example, [2-10]. During the last years, electron interference effects have been widely investigated in electron- and ion-induced ionization [7-18], as well as in photoionization [19-27] of isolated atoms and molecules. In the electron interference experiments $[5-15,19-23]$, each single electron hits the position-sensitive detector like a particle but traverses the interferometer slits (or scatters on atomic centers) like a wave. Thus, over many repetitions, an interference pattern builds up as oscillations of the intensity $I\left(\theta_{d}\right)=\int(d I / d \lambda) d \lambda$ with the observation angle $\theta_{d}[5-10]$. In experiments for which the actual wavelength distribution $d I / d \lambda$ can be characterized by a well-defined width at half maximum $\Delta \lambda$, the question arises whether it would be possible to observe similar oscillations in quantities other than $I\left(\theta_{d}\right)$, as for instance the linewidth $\Delta \lambda$ itself.
Recently, we studied the process:

$$
\mathrm{He}^{2+}+\mathrm{H}_{2}\left(1 \sigma_{g}\right) \longrightarrow \mathrm{He}^{* *}\left(2 \ell n \ell^{\prime}, n \geq 2\right)+\mathrm{H}^{+}+\mathrm{H}^{+}
$$

where the outgoing autoionizing helium atom plays the role of the source of a single electron emitted with a wavelength $\lambda$ of the order of a few Angstroms, while the two residual protons provide the double-center interferometer [7-10]. First, investigation of the total intensity of undiscerned $2 \ell n \ell^{\prime}$ $(n \geq 2)$ autoionization configurations revealed oscillations in the angular distribution of the scattered electrons, showing that each electron interferes with itself [7].

More recently, instead of following the standard procedure of seeking for oscillations on the line intensity, we concentrated on studying the linewidth [10]. This approach seems to have never been tried before either with electrons or photons. We focused on the single $2 s^{2}{ }^{1} S$ line and determined its maximum $I_{\max }$ and linewidth $\Delta \lambda$ as a function of the detection angle. Figure 1 shows the oscillating terms of $I_{\max }$ and $\Delta \lambda$ obtained in both cases by dividing the experimental data by a monotonic background. The maximum $I_{\max }$ of $d I / d \lambda$ oscillates in phase with the intensity $I\left(\theta_{d}\right)[10]$. On 


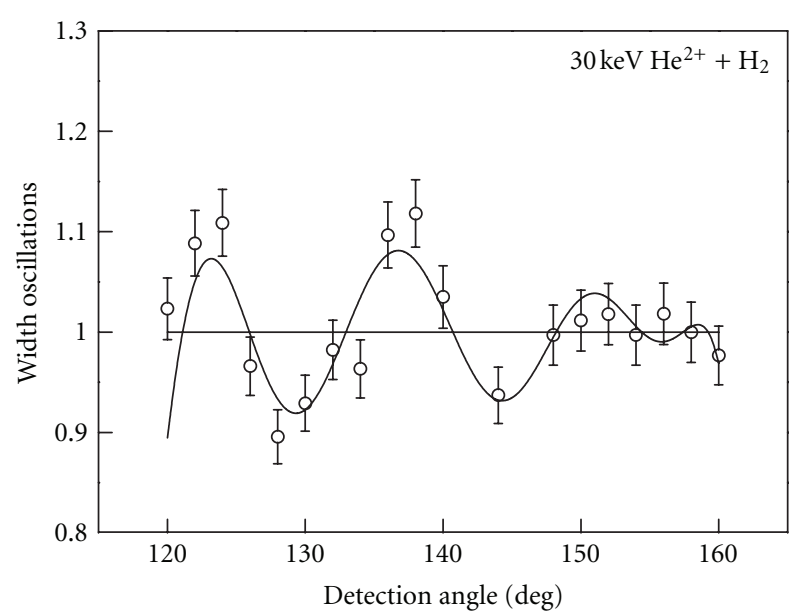

(a)

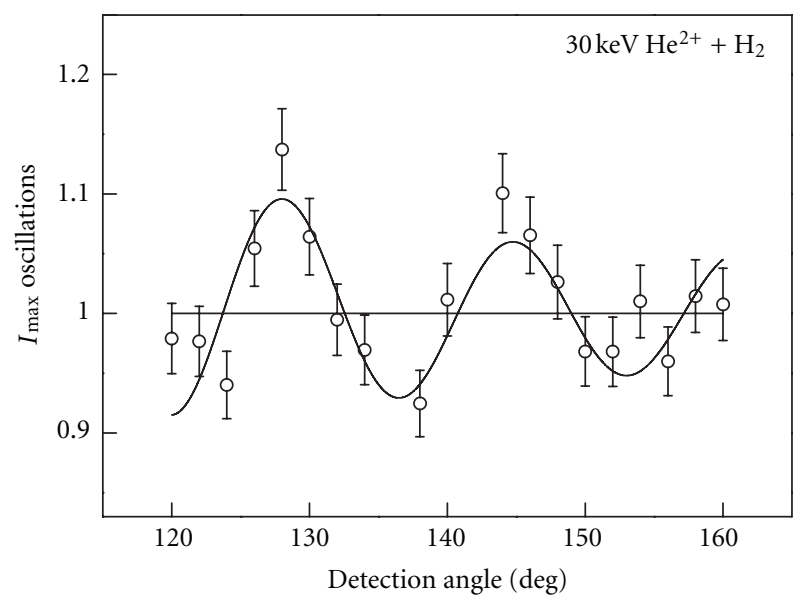

(b)

Figure 1: Oscillating term in the experimental width and maximum intensity of the $2 s^{2}{ }^{1} S$ line in $30-\mathrm{keV}^{3} \mathrm{He}^{2+}+\mathrm{H}_{2}$ collisions as a function of the detection angle. The full curves fit the experimental results, using the Bessel function of zero and first order.

the other hand, the $2 s^{2}{ }^{1} S$ linewidth was found to strongly oscillate in counter phase with the maximum (Figure 1), a fact that can be explained by means of simple theoretical arguments [10]. These results provided a new demonstration of the Young-type interference of single electrons.

The linewidth oscillations reported in our previous work [10] might be prone to be found in similar configurations with matter particles and photons. The question arises whether the analogy between photon and electron interferences can be extended to quantities such as the linewidth. Thus, in the present work, we revisited the photon interference experiment in order to determine the wavelength distribution as a function of the detection position in the interference pattern.

\section{Experimental Setup}

The optical setup is based on the classical Lloyd mirror experiment, first described by Lloyd in 1834. For alignment sim-

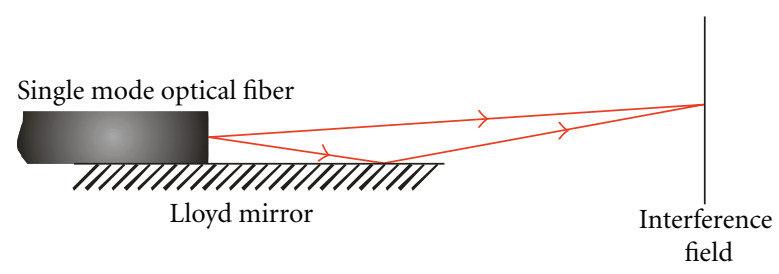

FIGURE 2: Schematic view of the experimental setup. Light originating from the optical fiber can reach the detector either directly or after reflection on the mirror.

plicity and robustness, the current system is an all-fiberized transposition to the original experiment. The light source is a superluminescent erbium-doped silica fiber (Figure 2).

The output power of the light source is typically of a few $\mathrm{mW}$ with a broadband optical emission spectrum between 1.53 and $1.56 \mu \mathrm{m}$. A peak centered at $\sim 1532 \mathrm{~nm}$ with a width of $\sim 0.93 \mathrm{~nm}$ is well separated from the rest of the emission spectrum. This emission peak is selected for the present optical experiment. The output facet of the optical fiber $(9 \mu \mathrm{m}$ fiber core diameter) acts as a high-brightness spatially coherent light source. A metal-coated plane mirror is precisely positioned at razing angle close to the optical fiber for creating a virtual light source. Moving the fiber allows adjusting the distance between the real and virtual light sources from $125 \mu \mathrm{m}$ up to $500 \mu \mathrm{m}$. The reflected light interferes with the direct light. The resulting interference fringes are detected point by point using a multimode fiber mounted on a step-motorized translation stage.

The entrance facet of the multimode fiber is located 30 to $50 \mathrm{~mm}$ away from the light source position. For each transverse position (moving step down to $20 \mu \mathrm{m}$ ), the collected light is analyzed using an optical spectrum analyzer (OSA) for simultaneous recording of the spectral and intensity distributions versus the detection position in the interference field.

\section{Results and Discussion}

The upper part of Figure 3 shows a typical experimental interference pattern, consisting in well-defined oscillations in the photon intensity distribution versus the position along a transverse direction with respect to the mirror.

The width of the fringes was found to be about $0.2 \mathrm{~mm}$. The middle part of the figure exhibits the spectral width of the interfering light as a function of the detection position. The average value of the width is $\sim 0.93 \mathrm{~nm}$. Whereas quasi-sinusoidal oscillations were observed for electrons, a nonsinusoidal but periodic dependency is visible for the width in case of photons. A significant change in $\Delta \lambda-$ typically larger than $0.01 \mathrm{~nm}$ - occurs at the position of dark fringes, while a smooth variation is observed for $\Delta \lambda$ when varying the detection position from a dark fringe to the next one. The same period is obtained for the intensity and the width. Lastly, the position $\lambda_{\max }$ of the maximum of $d I / d \lambda$ is presented in the bottom part of Figure 3. The dependency of $\lambda_{\max }$ versus the detection position is found to be similar to 


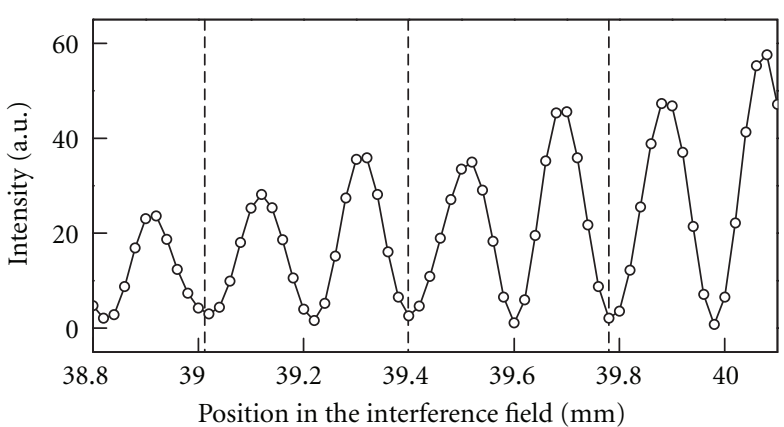

(a)

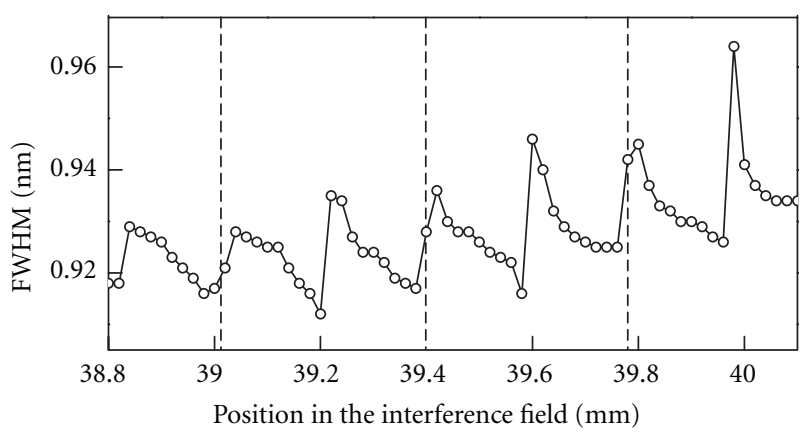

(b)

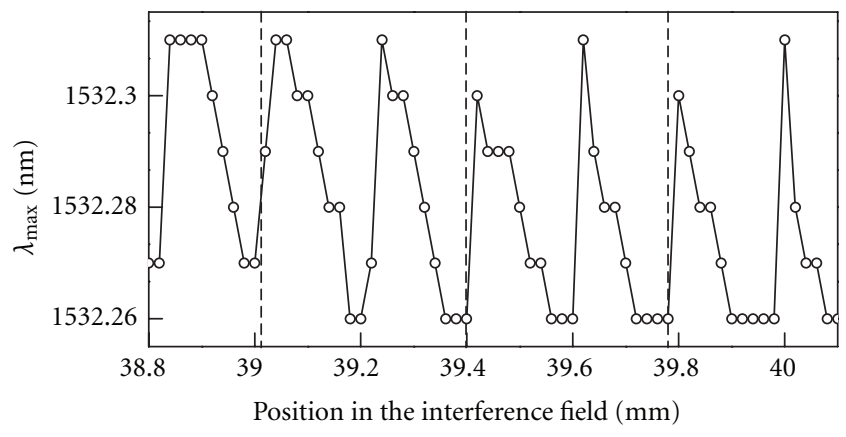

(c)

FIGURE 3: Experimental results: total light intensity $I\left(\theta_{d}\right)$ (top), full width at half maximum $\Delta \lambda$ (middle), and position $\lambda_{\max }$ (bottom) of the spectral distribution $d I / d \lambda$ as a function of the position along a direction perpendicular to the Lloyd mirror. The error bars (not shown) are of about $0.01 \mathrm{~nm}$ for $\lambda_{\max }$, while they are smaller than $0.01 \mathrm{~nm}$ for $\Delta \lambda$.

that of $\Delta \lambda$. When determining $\lambda_{\max }$, we considerably reduced the time of analysis by scanning the spectra in a restricted range of wavelengths. Nevertheless, although truncated, the obtained results (bottom of Figure 3) were accurate enough to reveal the periodic variation of $\lambda_{\max }$ with respect to the detection position in the interference field.

The present experiment shows that interference phenomena with photons manifest themselves not only as a sinusoidal variation of the light intensity versus the position in the interference pattern but also as a periodic position dependency of both $\lambda_{\max }$ and $\Delta \lambda$. A simple theoretical approach based on classical optics reproduces fairly well the main experimental features (Figure 4). Our present findings

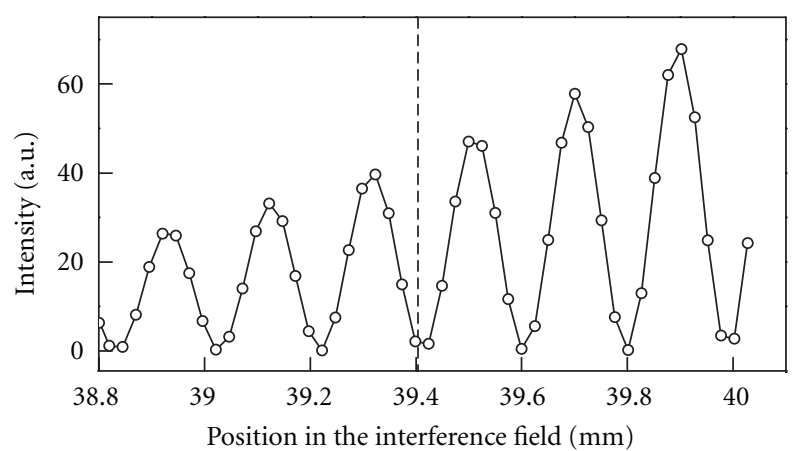

(a)

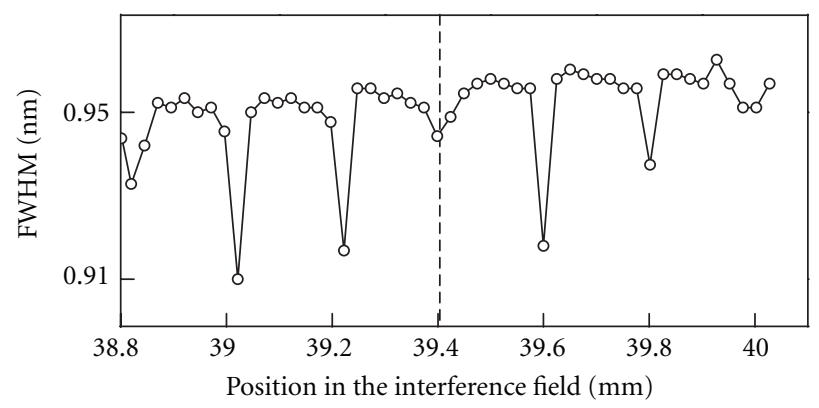

(b)

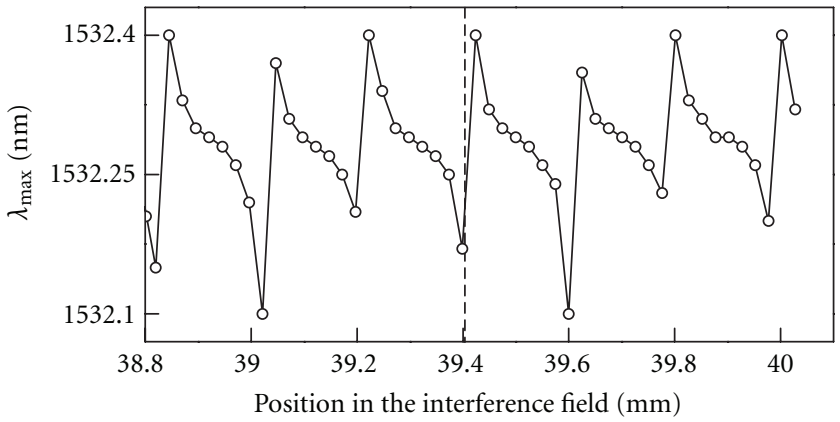

(c)

FIgURE 4: Calculation results: total light intensity $I\left(\theta_{d}\right)$ (top), full width at half maximum $\Delta \lambda$ (middle), and position $\lambda_{\max }$ (bottom) of the spectral distribution $d I / d \lambda$ as a function of the position along a direction perpendicular to the Lloyd mirror.

with photons provide a qualitative support to our previous interpretation of the oscillating behavior of the autoionization linewidth as a signature of a Young-type electron interference effect.

\section{Conclusion}

Photon experiments have been conducted using a Lloyd mirror and a $1.5 \mu \mathrm{m}$ source. In addition to well-defined oscillations in the intensity distribution, the width and the maximum position of the wavelength distribution present a periodic nonsinusoidal dependency versus the detection position in the interference field.

The present results extend the qualitative analogy between photon and electron interference experiments. The 
observed differences between photon and electron interferences can be attributed to the different nature of the interaction involved in each case, since the interaction between the photon and the interferometer is well localized, whereas the Coulombic interaction between the electron and the protons has a long-range character.

\section{Acknowledgments}

This work was supported by the French-Argentine Cooperation Program within the CNRS-CONICET Agreement 2011, the ECOS-Mincyt Collaboration Agreement (Project A07E04), the Agencia National de Promoción Científica y Tecnológica (Grant 03-20548), the Consejo Nacional de Investigaciones Cientificas y Tecnicas (Grant PIP 112-20080101269), and the Universidad Nacional de Cuyo (Grant 06/C340), Argentina.

\section{References}

[1] L. de Broglie, "Ondes et quanta," Comptes Rendus de l'Académie des Sciences, vol. 177, pp. 507-510, 1923.

[2] C. J. Davisson, "Are electrons waves?" Journal of the Franklin Institute, vol. 205, no. 5, pp. 597-623, 1928.

[3] C. Jönsson, "Elektroneninterferenzen an mehreren künstlich hergestellten Feinspalten," Zeitschrift für Physik, vol. 161, no. 4, pp. 454-474, 1961.

[4] D. Brandt, "Electron diffractionat multiple slits," American Journal of Physics, vol. 42, no. 1, 4 pages, 1974.

[5] P. G. Merli, G. F. Missiroli, and G. Pozzi, "On the statistical aspect of electron interference phenomena," American Journal of Physics, vol. 44, no. 3, pp. 306-307, 1976.

[6] A. Tonomura, J. Endo, T. Matsuda, T. Kawasaki, and H. Ezawa, "Demonstration of single-electron buildup of an interference pattern," American Journal of Physics, vol. 57, no. 2, pp. 117$120,1989$.

[7] J.-Y. Chesnel, A. Hajaji, R. O. Barrachina, and F. Frémont, "Young-type experiment using a single-electron source and an independent atomic-size two-center interferometer," Physical Review Letters, vol. 98, no. 10, Article ID 100403, 4 pages, 2007.

[8] F. Frémont, D. Gruyer, V. Helaine et al., "A young doubleslit experiment using a single electron source," in Handbook of Interferometers: Research, Technology and Applications, D. Halsey and W. Raynor, Eds., Nova Science, Hauppauge, NY, USA, 2009.

[9] F. Frémont, S. Suarez, R. O. Barrachina et al., "Young-type interferences using single-electron sources and an atomic-size two-center interferometer: dependence with interferometer parameters," Nuclear Instruments and Methods in Physics Research B, vol. 267, no. 2, pp. 206-210, 2009.

[10] R. O. Barrachina, F. Frémont, K. Fossez et al., "Linewidth oscillations in a nanometer-size double-slit interference experiment with single electrons," Physical Review A, vol. 81, no. 6, Article ID 060702(R), 2010.

[11] N. Stolterfoht, B. Sulik, V. Hoffmann et al., "Evidence for interference effects in electron emission from $\mathrm{H}_{2}$ colliding with $60 \mathrm{MeV} / \mathrm{u} \mathrm{Kr}{ }^{3++}$ ions," Physical Review Letters, vol. 87, no. 2, Article ID 023201, 4 pages, 2001.

[12] J. A. Tanis, J.-Y. Chesnel, B. Sulik et al., "Angular and highfrequency analysis of electron interference structures in $60 \mathrm{MeVu} \mathrm{Kr}{ }^{34+}+\mathrm{H}_{2}$ collisions," Physical Review A, vol. 74, no. 2, Article ID 022707, 6 pages, 2006.
[13] D. Fischer, M. Gudmundsson, K. Støchkel et al., "Two-center interference in $\mathrm{p}-\mathrm{H}_{2}$ electron-transfer collisions," Journal of Physics, vol. 88, no. 1, Article ID 012021, 2007.

[14] D. Misra, U. Kadhane, Y. P. Singh, L. C. Tribedi, P. D. Fainstein, and P. Richard, "Interference effect in electron emission in heavy ion collisions with $\mathrm{H}_{2}$ detected by comparison with the measured electron spectrum from atomic hydrogen," Physical Review Letters, vol. 92, no. 15, Article ID 153201, 4 pages, 2004.

[15] L. R. Hargreaves, C. Colyer, M. A. Stevenson et al., "(e,2e) study of two-center interference effects in the ionization of $\mathrm{N}_{2}$," Physical Review A, vol. 80, no. 6, Article ID 062704, 7 pages, 2009.

[16] F. Frémont, A. Hajaji, A. Naja et al., "Fast oscillating structures in electron spectra following $\mathrm{He}^{\mathrm{q}+}+\mathrm{He}$ collisions $(\mathrm{q}=1,2)$ at low projectile energies," Physical Review A, vol. 72, no. 5, Article ID 050704(R), 4 pages, 2005.

[17] L. Nagy, L. Kocbach, K. Póra, and J. P. Hansen, "Interference effects in the ionization of $\mathrm{H}_{2}$ by fast charged projectiles," Journal of Physics B, vol. 35, no. 20, p. L453, 2002.

[18] M. E. Galassi, R. D. Rivarola, P. D. Fainstein, and N. Stolterfoht, "Young-type interference patterns in electron emission spectra produced by impact of swift ions on $\mathrm{H}_{2}$ molecules," Physical Review A, vol. 66, no. 5, Article ID 052705, 4 pages, 2002.

[19] D. Akoury, K. Kreidi, T. Jahnke et al., "The simplest double slit: interference and entanglement in double photoionization of $\mathrm{H}_{2}$," Science, vol. 318, no. 5852, pp. 949-952, 2007.

[20] M. Meckel, D. Comtois, D. Zeidler et al., "Laser-induced electron tunneling and diffraction," Science, vol. 320, no. 5882, pp. 1478-1482, 2008.

[21] M. S. Schöffler, K. Kreidi, D. Akoury et al., "Photo-doubleionization of $\mathrm{H}_{2}$ : two-center interference and its dependence on the internuclear distance," Physical Review A, vol. 78, no. 1, Article ID 013414, 5 pages, 2008.

[22] M. Okunishi, R. Itaya, K. Shimada et al., "Two-source double-slit interference in angle-resolved high-energy abovethreshold ionization spectra of diatoms," Physical Review Letters, vol. 103, no. 4, Article ID 043001, 4 pages, 2009.

[23] S. E. Canton, E. Plésiat, J. D. Bozek et al., "Direct observation of Young's double-slit interferences in vibrationally resolved photoionization of diatomic molecules," Proceedings of the National Academy of Sciences, vol. 108, no. 18, pp. 7302-7306, 2011.

[24] S. N. Yurchenko, S. Patchkovskii, I. V. Litvinyuk, P. B. Corkum, and G. L. Yudin, "Laser-induced interference, focusing, and diffraction of rescattering molecular photoelectrons," Physical Review Letters, vol. 93, no. 22, Article ID 223003, 4 pages, 2004.

[25] X. J. Liu, N. A. Cherepkov, S. K. Semenov et al., "Young's double-slit experiment using core-level photoemission from $\mathrm{N}_{2}$ : revisiting Cohen-Fano's two-centre interference phenomenon," Journal of Physics B, vol. 39, no. 23, pp. 4801-4818, 2006.

[26] J. Fernández, O. Fojón, A. Palacios, and F. Martín, "Interferences from fast electron emission in molecular photoionization," Physical Review Letters, vol. 98, no. 4, Article ID 043005, 4 pages, 2007.

[27] U. Becker, "Molecular physics: matter-wave interference made clear," Nature, vol. 474, pp. 586-587, 2011. 


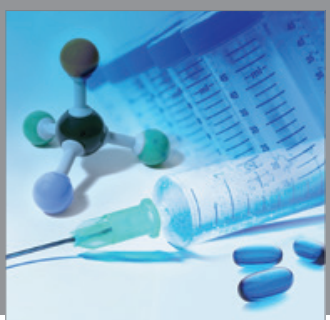

International Journal of

Medicinal Chemistry

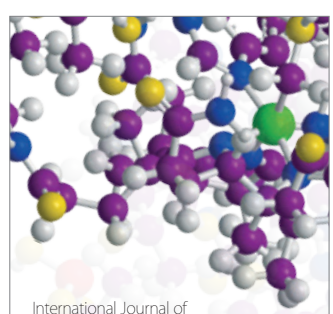

Carbohydrate Chemistry

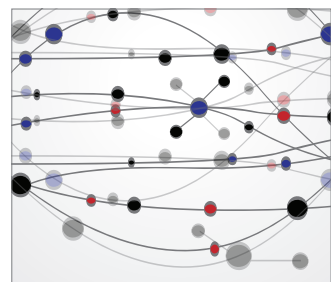

The Scientific World Journal
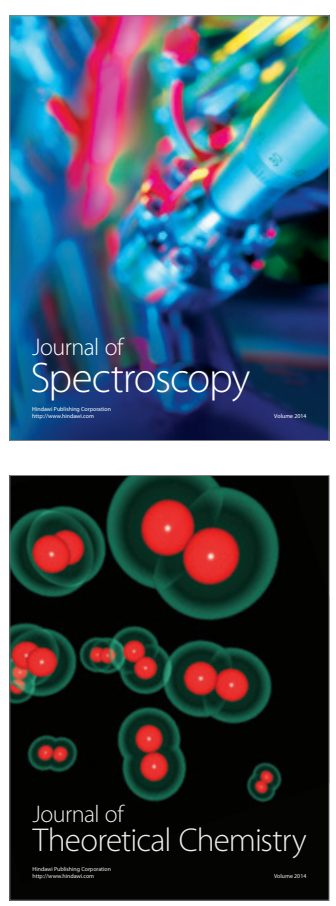
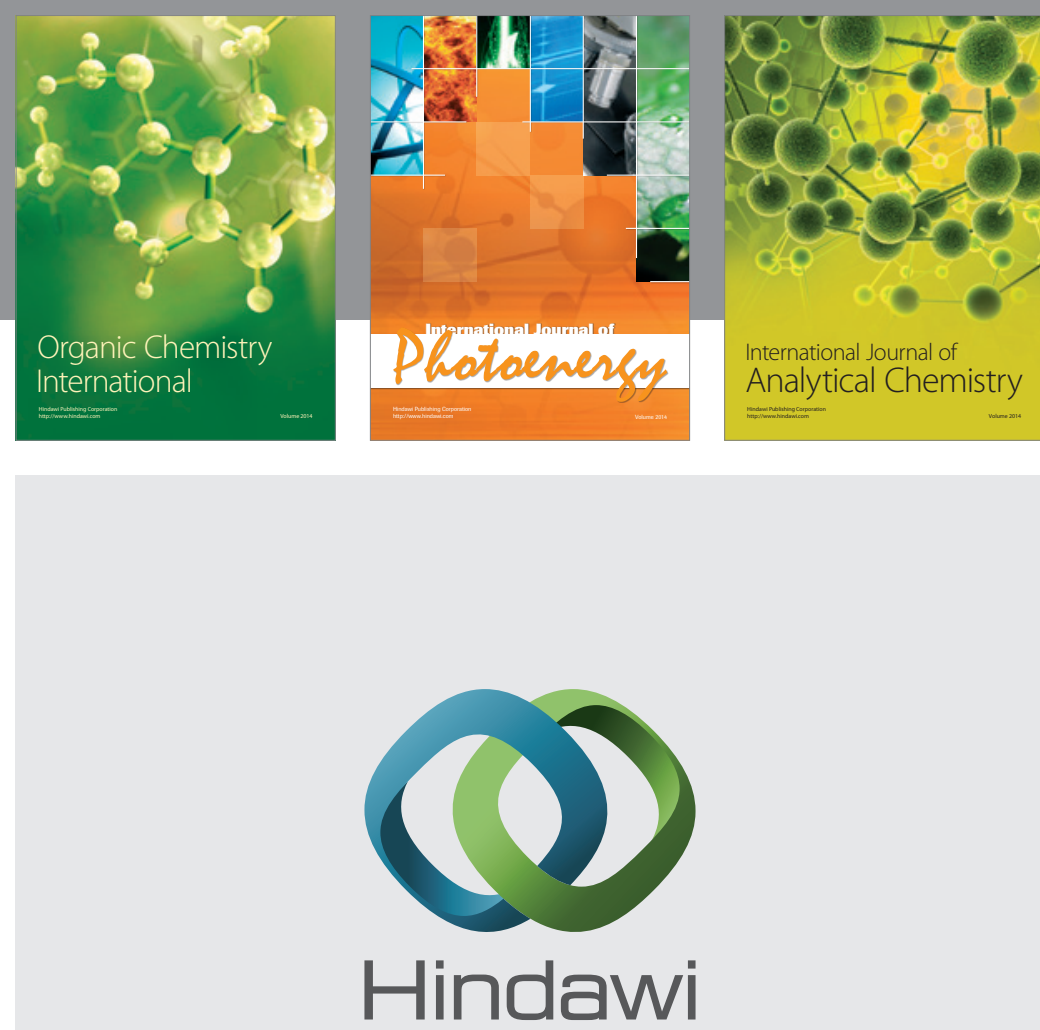

Submit your manuscripts at

http://www.hindawi.com
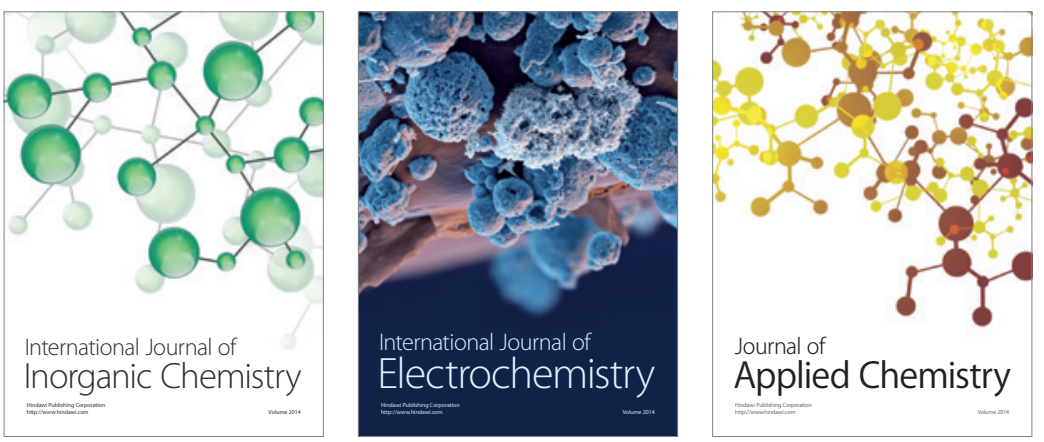

Journal of

Applied Chemistry
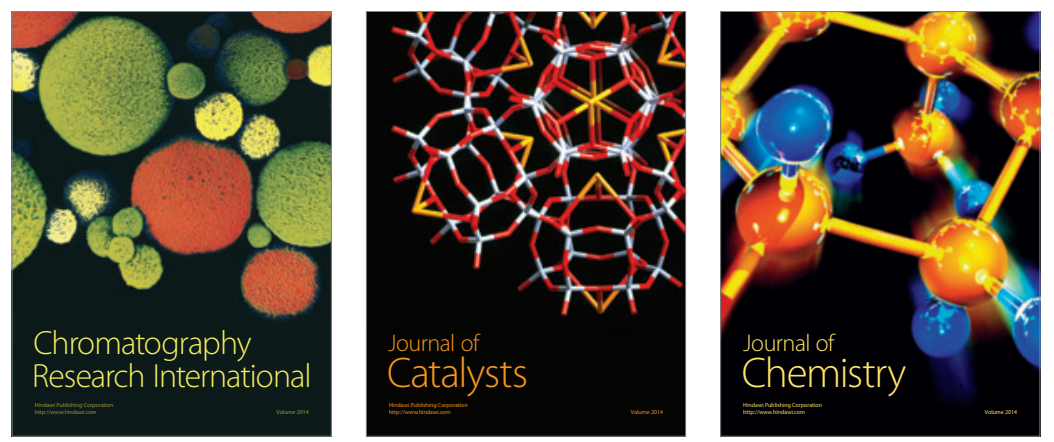
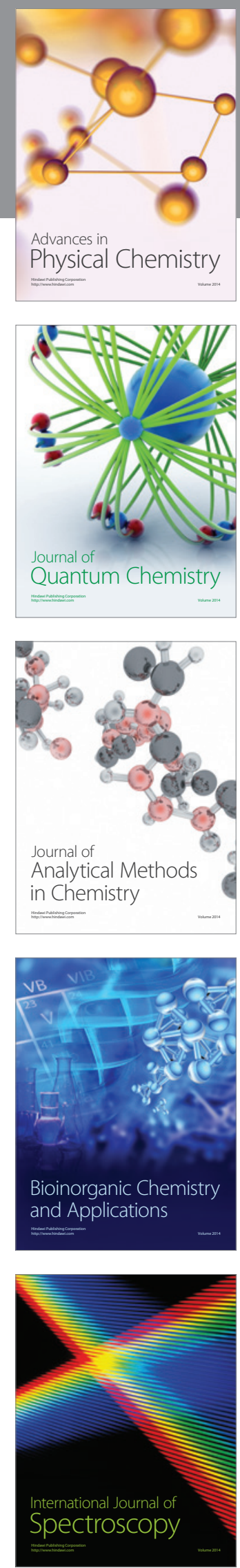